\title{
3D Interdigitated Vertically Aligned Carbon Nanotube Electrodes for Electrochemical Impedimetric Biosensing
}

\author{
Benjamin J. Brownlee \\ Brigham Young University - Provo \\ Jonathan C. Claussen \\ lowa State University \\ Brian D. Iverson \\ Brigham Young University - Provo, bdiverson@byu.edu
}

Follow this and additional works at: https://scholarsarchive.byu.edu/facpub

Part of the Mechanical Engineering Commons

\section{Original Publication Citation}

Brownlee, B. J., Claussen, J. C., and Iverson, B. D., 2020, “3D Interdigitated vertically aligned carbon nanotube electrodes for electrochemical impedimetric biosensing," ACS Applied Nano Materials, 3, p. 10166-10175. DOI: 10.1021/acsanm.0c02121

\section{BYU ScholarsArchive Citation}

Brownlee, Benjamin J.; Claussen, Jonathan C.; and Iverson, Brian D., "3D Interdigitated Vertically Aligned Carbon Nanotube Electrodes for Electrochemical Impedimetric Biosensing" (2020). Faculty Publications. 5089.

https://scholarsarchive.byu.edu/facpub/5089

This Peer-Reviewed Article is brought to you for free and open access by BYU ScholarsArchive. It has been accepted for inclusion in Faculty Publications by an authorized administrator of BYU ScholarsArchive. For more information, please contact ellen_amatangelo@byu.edu. 


\title{
3D Interdigitated Vertically Aligned Carbon Nanotube Electrodes for Electrochemical Impedimetric Biosensing
}

\author{
Benjamin J. Brownlee, Jonathan C. Claussen, and Brian D. Iverson*
}

Cite This: ACS Appl. Nano Mater. 2020, 3, 10166-10175

Read Online

ABSTRACT: Advances in nanomaterials, combined with electrochemical impedance spectroscopy (EIS), have allowed electrochemical biosensors to have high sensitivity while remaining labelfree, enabling the potential for portable diagnosis at the point-ofcare. We report porous, 3D vertically aligned carbon nanotube (VACNT) electrodes with underlying chromium electrical leads for impedance-based biosensing. The electrodes are characterized by electrode height $(5,25$, and $80 \mu \mathrm{m})$, gap width $(15$ and $25 \mu \mathrm{m})$, and geometry (interdigitated and serpentine) using scanning electron microscopy, cyclic voltammetry, and EIS. The protein streptavidin is functionalized onto VACNT electrodes for detection of biotin, as confirmed by fluorescence microscopy.
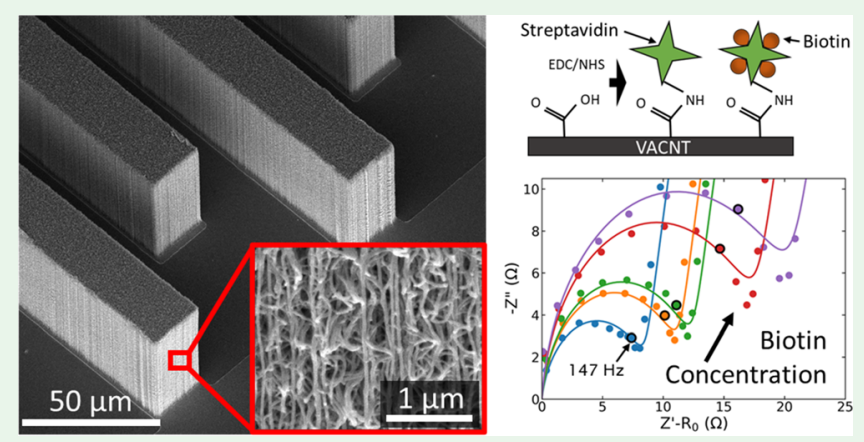

EIS is used to measure the change in impedance across electrodes for different biotin concentrations. The impedance data show two distinct semicircular regions, which are modeled by an equivalent electrical circuit. VACNT electrode height, gap width, and geometrical pattern each have an impact on sensor sensitivity, with tall, closely spaced VACNT interdigitated electrodes (IDEs) having the highest sensitivity. With an electroactive surface area that is 15 times the $2 \mathrm{D}$ geometric area, $80 \mu \mathrm{m}$ tall VACNT IDEs with a gap width of $15 \mu \mathrm{m}$ are 4.3 times more sensitive than short $(5 \mu \mathrm{m})$ IDEs and 1.6 times more sensitive than serpentine electrodes. The biosensors obtain a limit of detection of $1 \mathrm{ng} / \mathrm{mL}$ biotin with two linear sensing regions $(0.001-1$ and $1-100 \mu \mathrm{g} /$ $\mathrm{mL}$ ). Although this biosensing platform is shown with streptavidin and biotin, it could be extended to other proteins, antibodies, viruses, and bacteria.

KEYWORDS: electrochemical sensor, interdigitated electrodes, carbon nanotubes, biosensor, streptavidin-biotin

\section{INTRODUCTION}

Carbon nanomaterials such as carbon nanotubes (CNTs), graphene, and graphene oxide have been shown to effectively increase the sensitivity of electrochemical sensors. ${ }^{1,2}$ Specifically, micro and nanostructured surfaces greatly increase the electrode surface area and may outperform smooth, planar geometries. ${ }^{3}$ The high electrical conductivity and high surface area of CNTs make them attractive as an electrode nanomaterial. ${ }^{4}$ Both single-walled and multi-walled CNTs are often incorporated into sensors by casting randomly dispersed CNTs on an electrode surface. ${ }^{5-7}$ Although dispersed CNTs allow for unique sensors such as paper-based CNT sensors or inkjet-printed CNT sensors, ${ }^{8-10}$ nanostructures grown from an electrode surface have been shown to have greater stability and allow for greater exposed surface area than randomly dispersed nanomaterials. ${ }^{11}$ Particularly, vertically aligned carbon nanotubes (VACNTs) provide an ordered, preferential orientation of CNTs that are grown from the substrate. Very high aspect ratio geometries are possible through patterning of the VACNT forest, offering the potential for three-dimensional (3D), porous electrodes that may be exploited for concentration measurement of proteins, antibodies, or other molecules. The high surface-area-to-volume ratio that comes from the 3D, porous VACNT electrodes enables higher protein capture capacity relative to planar electrodes, leading to the potential for improved sensitivity. ${ }^{12,13}$ High sensor sensitivity is important for enabling detection of low concentrations of a target analyte, opening the door for new sensing applications, such as early diagnostics.

Advances in electrochemical biosensors have pushed toward the ability to obtain effective, rapid results. While biosensors can use a variety of detection methods such as optical, electrical, or piezoelectric, electrochemical sensors offer the advantage of being repeatable, affordable, and easy-to-use. ${ }^{14}$ In particular, electrochemical sensors comprised of interdigitated electrodes (IDE) exhibit low ohmic drops, high signal-to-noise ratios, and fast response times. ${ }^{15}$ IDE biosensors are often

Received: August 3, 2020

Accepted: September 29, 2020

Published: September 29, 2020 
impedimetric, using electrochemical impedance spectroscopy (EIS) to measure small changes in impedance at an electrode surface and offer the potential for label-free and real-time detection of various analytes. ${ }^{16,17}$ These advantages of electrochemical IDE biosensors enable portable diagnosis at the point-of-care by eliminating the need for laboratory expertise and the time associated with conventional immunoassay-based testing. ${ }^{18,19}$

While IDE geometries are typically used, a serpentine electrode (SE) design has been shown to increase sensitivity of a capacitive humidity sensor when compared to an IDE of the same material and dimensions. ${ }^{20}$ A serpentine design has been used in other applications, such as in the design of a flow field for redox flow batteries to enhance performance through geometry. ${ }^{21}$ Impedance-based serpentine sensors for the detection of biorecognition agents do not appear in the literature, and it would be advantageous to determine if the serpentine geometry can improve sensitivity similar to the capacitive humidity sensor. Hence, both the IDE and serpentine geometry would be of interest to explore in terms of electrochemical sensing performance.

In addition to electrode geometry, the electrode surface area also plays an important role in electrochemical sensing. Typical planar electrodes have a thickness that is on the order from 10 's to 100 's of nanometers. While an electrode height (thickness) of a few micrometers may be considered 3D, patterned VACNTs offer the potential to increase the electrode thicknesses to greater than 100's of micrometers. VACNTs have previously been used as IDEs for capacitors with VACNT heights ranging from 34 to $70 \mu \mathrm{m}$ with a $20-35$ $\mu \mathrm{m}$ gap between electrodes. ${ }^{22-24}$ Although such tall heights are not typically reported for electrochemical biosensors, our previous work with an oral cancer biomarker had a VACNT electrode height of about $75 \mu \mathrm{m}$ and gap of $25 \mu \mathrm{m} .{ }^{13}$ Smaller gap width between tall VACNT electrodes may likely improve sensitivity, a relationship that has previously been shown with planar electrodes. ${ }^{25}$

Previous IDE sensors were made entirely of VACNTs, including the interdigitated fingers and the electrical leads. ${ }^{13} \mathrm{~A}$ redox sensor for dopamine was constructed with a VACNT electrode height of 0.66 and $3 \mu \mathrm{m}$ gap, where the fingers of VACNT IDEs were grown directly on metallic electrical leads. ${ }^{26}$ Others have shown that an electrical connection can be made from VACNTs through an insulating aluminum oxide layer to an underlying metallic electrical lead. ${ }^{27}$ This approach using a thin metallic electrical lead offers a way to separate the leads from the sensing region and enables access to the sensor when used within a microfluidic cavity. The ability to use a sensor in a microfluidic channel is an additional feature that promotes point-of-care diagnosis, as microfluidic-based detection offers a simple, fast, and convenient platform relative to traditional macroscopic platforms. ${ }^{28}$

This work characterizes porous, 3D VACNT electrodes for impedance-based biosensing. These unique structures provide very high surface area electrodes for which the nanomaterial height is significantly taller than traditional planar electrodes. An application of these VACNT electrodes has been successfully shown for the detection of oral cancer biomarkers for a single electrode geometry (75 $\mu \mathrm{m}$ tall IDE with $25 \mu \mathrm{m}$ gap width). ${ }^{13}$ This work performs a parameter study to determine the impact of VACNT height, gap width, and electrode geometry pattern (IDE vs SE) on the sensitivity of VACNT biosensors. The topic of electrode height is emphasized because the trend of gap width has previously been explored for planar electrodes. ${ }^{25}$ The previous oral cancer sensor was made of all VACNT, but here, an underlying chromium layer is used for electrical leads to improve stability, reduce internal resistance, and provide a potential path for integration with a microfluidic channel. Patterned VACNT forests were grown to heights of 5, 25, and $80 \mu \mathrm{m}$ on underlying chromium electrical leads and then coated with amorphous carbon to provide additional rigidity while still maintaining porosity. To compare the performance of the distinctly constructed VACNTs within an electrochemical biosensing paradigm, the common protein-ligand system (streptavidin-biotin) was employed. The protein streptavidin was functionalized onto VACNT electrodes for detection of biotin, where biotin binds to streptavidin in one of the strongest known noncovalent bonds between a protein and a ligand. $^{29}$ Fluorescence microscopy aided in confirming streptavidin and biotin binding on VACNTs. Electrical impedance spectroscopy (EIS) was used to measure the change in impedance across electrodes for different biotin concentrations. The change in impedance with the addition of streptavidin and biotin was modeled by an equivalent electrical circuit, and the change in imaginary impedance at a single frequency was monitored for different biotin concentrations. VACNT height and electrode geometry are shown to influence biosensor sensitivity, with tall, closely spaced IDEs having the highest sensitivity. Comparison between 3D IDE and SE configurations are also provided. Although this biosensing platform is shown with streptavidin and biotin, it could be extended to other proteins, antibodies, viruses, and bacteria by functionalizing with another biorecognition agent with a selective binding mechanism.

\section{EXPERIMENTAL SECTION}

2.1. Electrode Fabrication. Photolithography was used to pattern positive photoresist (AZ3330) on a $100 \mathrm{~mm}$ diameter, oxidized silicon ( $\mathrm{Si}$ ) wafer (1 $\mu \mathrm{m}$ thick oxide). A patterned photoresist was used to create the electrode designs and the $\mathrm{Cr}$ electrical leads for each electrode $(13 \times 0.5 \mathrm{~mm})$. A $75 \mathrm{~nm}$ layer of $\mathrm{Cr}$ was deposited onto the patterned photoresist by electron beam evaporation. A lift-off process was performed by sonication in $\mathrm{N}$ methyl-2-pyrrolidone (NMP) for $30 \mathrm{~min}$, leaving only patterned $\mathrm{Cr}$ on the oxidized wafer. In this manner, $\mathrm{Cr}$ was deposited below all $3 \mathrm{D}$ electrode regions and as electrical leads to the sensor electrodes. After covering the contact pads to the electrical leads with a permanent marker, $150 \mathrm{~nm}$ of aluminum oxide $\left(\mathrm{Al}_{2} \mathrm{O}_{3}\right)$ was deposited by electron beam evaporation. NMP sonication was performed for $5 \mathrm{~min}$ to expose the contact pads by removing the marker ink and $\mathrm{Al}_{2} \mathrm{O}_{3}$ for electrical connectors. A second photolithography process was used to cover the wafer in photoresist except in regions where sensor fingers are to be constructed. A7 $\mathrm{nm}$ film of iron $(\mathrm{Fe})$ was thermally evaporated onto the patterned photoresist, followed by sonication in $\mathrm{NMP}$ for $15 \mathrm{~min}$, leaving patterned $\mathrm{Fe}$ on a portion of the $\mathrm{Al}_{2} \mathrm{O}_{3} / \mathrm{Cr}$ layer. In this manner, the catalyst for CNT growth was patterned so that growth would only occur in the $3 \mathrm{D}$ electrode regions. The patterned wafer was diced into $16.1 \times 8 \mathrm{~mm}$ rectangles using a dicing saw with a diamond-coated blade.

VACNT growth methods were similar to previously published protocols. ${ }^{30,31}$ VACNTs were grown by chemical vapor deposition (CVD) in a $1 \mathrm{in}$. diameter Lindberg/Blue $M$ tube furnace with flowing hydrogen $\left(\mathrm{H}_{2}, 311 \mathrm{sccm}\right)$ and ethylene $\left(\mathrm{C}_{2} \mathrm{H}_{4}, 338 \mathrm{sccm}\right)$ at $750{ }^{\circ} \mathrm{C}$ for 5,30 , or $120 \mathrm{~s}$. The temperature was then increased to 900 ${ }^{\circ} \mathrm{C}$, and the $\mathrm{C}_{2} \mathrm{H}_{4}$ flow rate was decreased to $193 \mathrm{sccm}$ to infiltrate (coat) the VACNTs with amorphous carbon for $60 \mathrm{~s}$. The electrodes were oxygen plasma etched $(250 \mathrm{~W}, 300 \mathrm{mTorr}, 30-60 \mathrm{~s})$ until the 
two electrodes were electrically isolated using a Technics Planar Etch II machine.

2.2. Electrochemical Measurements. All electrochemical experiments were performed using a $\mathrm{CH}$ Instruments (CHI) $660 \mathrm{E}$ potentiostat/galvanostat in a two-electrode configuration where one VACNT electrode was the working electrode, and the other VACNT electrode was the counter/reference electrode. The $\mathrm{Cr}$ contact pads were contacted by micromanipulation probes. The electrolyte was a static $50 \mu \mathrm{L}$ droplet of $5 \mathrm{mM}$ potassium ferricyanide $\left(\mathrm{K}_{3}\left[\mathrm{Fe}(\mathrm{CN})_{6}\right]\right)$, $5 \mathrm{mM}$ potassium ferrocyanide $\left(\mathrm{K}_{4}\left[\mathrm{Fe}(\mathrm{CN})_{6}\right]\right)$, and $100 \mathrm{mM} \mathrm{KCl}$ in phosphate-buffered saline (PBS, 1X, pH 7.4, Fisher Scientific). Cyclic voltammetry $(\mathrm{CV})$ was performed between -600 and $600 \mathrm{mV}$ relative to the VACNT electrode at scan rates from 150 to $1250 \mathrm{mV} / \mathrm{s}$. Ohmic drop correction was used to account for the resistance within the $\mathrm{Cr}$ electrical leads (about $300 \Omega$ ). The frequencies for electrochemical impedance spectroscopy (EIS) ranged from 10000 to $0.1 \mathrm{~Hz}$, with an input amplitude of $10 \mathrm{mV}$.

2.3. Streptavidin Functionalization. The carbon-infiltrated VACNT electrodes with exposed carboxyl groups $(\mathrm{COOH}$, see Scheme 1A) were incubated in a $50 \mu \mathrm{L}$ droplet of $100 \mathrm{mM} \mathrm{EDC}$ and

Scheme 1. Schematic of Binding Chemistry for VACNT Sensors $^{a}$

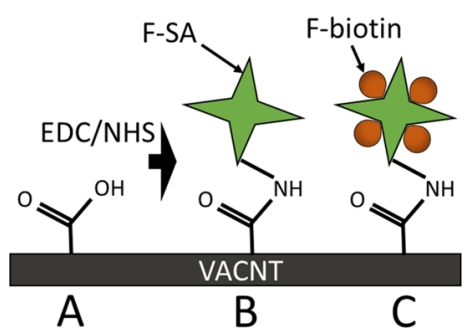

${ }^{a}$ (A) Carboxyl groups $(\mathrm{COOH})$ from carbon-infiltrated VACNTs. (B) F-SA (streptavidin) covalently bound to VACNTs using EDC/ NHS chemistry. (C) F-biotin bound to streptavidin.

$100 \mathrm{mM}$ NHS in 0.1 M 2-( $N$-morpholino) ethanesulfonic acid (MES, $\mathrm{pH}$ 4.7, Thermo Scientific 28 390) for $60 \mathrm{~min}$. The VACNT electrodes were then rinsed and soaked in PBS for $20 \mathrm{~min}$. A $20 \mu \mathrm{L}$ droplet of $500 \mu \mathrm{g} / \mathrm{mL}$, fluorescently tagged Atto 425 streptavidin (FSA, Sigma-Aldrich 09260) in PBS was placed on the EDC/NHSactivated VACNTs for $60 \mathrm{~min}$ to bind the SA protein to the VACNTs (see Scheme 1B). Electrodes were then rinsed and allowed to soak in PBS for $40 \mathrm{~min}$. While the stock F-SA solution was stored at $4{ }^{\circ} \mathrm{C}$, all functionalization steps and soakings were performed at room temperature.

2.4. Biotin Sensing. A $20 \mu \mathrm{L}$ droplet of $1 \mathrm{ng} / \mathrm{mL}$ fluorescently tagged Atto 565 biotin (F-biotin, Sigma-Aldrich 92 637) in PBS was placed on F-SA/VACNT sensors for $20 \mathrm{~min}$. Up to four F-biotins can bind to a single F-SA protein, as shown schematically in Scheme 1C. The sensor was then rinsed and allowed to soak in $50 \mu \mathrm{L}$ of PBS for 1 min after which electrochemical measurements were taken. The concentration of F-biotin was incrementally increased for each measurement following the same procedure above for each concentration $(0.01,0.1,1,10$, and $100 \mu \mathrm{g} / \mathrm{mL})$.

2.5. Fluorescence. Fluorescence measurements were performed using an Olympus FluoView FV1000 confocal laser scanning microscope. Wavelengths of 405 and $543 \mathrm{~nm}$ were used to excite the F-SA and F-biotin, respectively. The two fluorescent markers have very little overlap in absorption and emission so that the intensity of each is nearly independent of the other. Specific microscope settings included: $20 \times$ magnification, $12 \%$ laser power, and $200 \mu \mathrm{m}$ aperture size.

\section{RESULTS AND DISCUSSION}

3.1. Electrode Design. The IDE design is illustrated in Figure 1A, and the SE design is shown in Figure 1B. Dark red
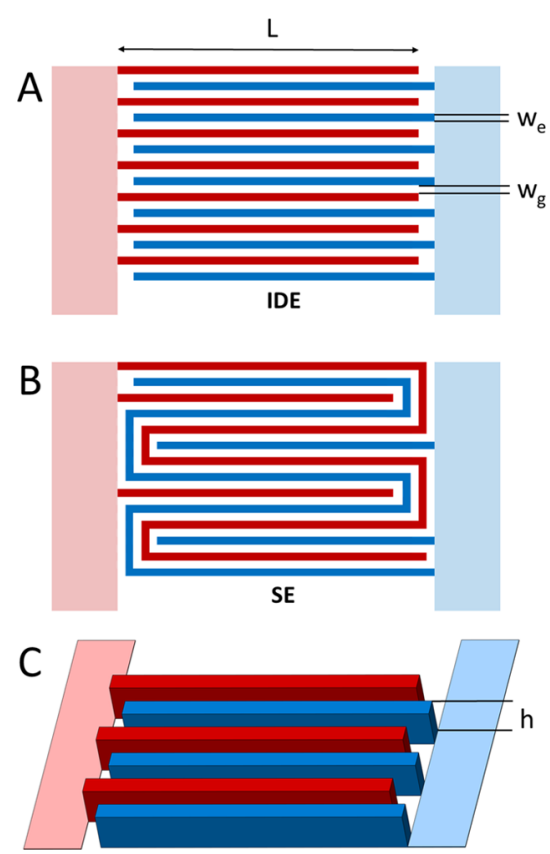

Figure 1. Schematic of the (A) IDE and (B) SE electrode arrangements with electrode length $(L)$, electrode width $\left(w_{\mathrm{e}}\right)$, and gap width $\left(w_{\mathrm{g}}\right)$ represented. Red and blue distinguish the different electrodes in the sensor, with the dark colors representing regions of VACNTs and light colors representing $\mathrm{Cr}$ leads under $\mathrm{Al}_{2} \mathrm{O}_{3}$. (C) Schematic emphasizing the 3D nature of VACNT IDE electrodes with electrode height $(h)$ represented. Images are not to scale and do not show all fingers.

and blue areas (central region) represent patterned forests of VACNTs acting as the electrodes; light red and blue areas (left and right edges) represent thin chromium $(\mathrm{Cr})$ electrical leads underneath an aluminum oxide $\left(\mathrm{Al}_{2} \mathrm{O}_{3}\right)$ layer. This approach with buried $\mathrm{Cr}$ electrical leads differs from our previously reported sensors ${ }^{13}$ with electrical leads comprised of VACNTs and allows for integration into a microfluidic channel (see Section 3.5 and the Supporting Information). Figure 1C illustrates the three-dimensional nature of VACNT IDE electrodes.

The electrode length $(L)$ was $2.95 \mathrm{~mm}$ for all sensors tested in this work. Two different electrode widths $\left(w_{\mathrm{e}}, 20\right.$ and 25 $\mu \mathrm{m})$ and two different gap widths ( $w_{\mathrm{g}}, 15$ and $\left.25 \mu \mathrm{m}\right)$ were used for IDE and SE sensor designs. VACNT height $(h)$ was varied for the IDE electrode arrangement $(5,25$, and $80 \mu \mathrm{m})$, resulting in six different geometries summarized in Table 1.

Each IDE sensor had 61 total fingers (30 and 31 per side) and each SE sensor occupied an equivalent area with two winding fingers ( 1 per side) and 20 shortened fingers ( 10 per side). For $w_{\mathrm{g}}=25 \mu \mathrm{m}$, the sensing region was nearly square at $3.025 \times 3 \mathrm{~mm}$, with a total electrode area of $4.5 \mathrm{~mm}^{2}$. For $w_{\mathrm{g}}=$

Table 1. Summary of Dimensions for Sensor Geometries

\begin{tabular}{cccc} 
geometry & $w_{\mathrm{e}}(\mu \mathrm{m})$ & $w_{\mathrm{g}}(\mu \mathrm{m})$ & $h(\mu \mathrm{m})$ \\
IDE & 25 & 25 & 5 \\
& 25 & 25 & 20 \\
& 25 & 25 & 80 \\
\multirow{2}{*}{ SE } & 20 & 15 & 80 \\
& 25 & 25 & 80 \\
& 20 & 15 & 80
\end{tabular}


$15 \mu \mathrm{m}$, the sensing region was smaller at $2.115 \times 3 \mathrm{~mm}$, with a total electrode area of $3.6 \mathrm{~mm}^{2}$. The two $\mathrm{Cr}$ electrical leads for each sensor are $13 \times 0.5 \mathrm{~mm}$.

3.2. VACNT Characterization. Figure $2 \mathrm{~A}$ shows the processing layers for biosensor fabrication, where an oxidized

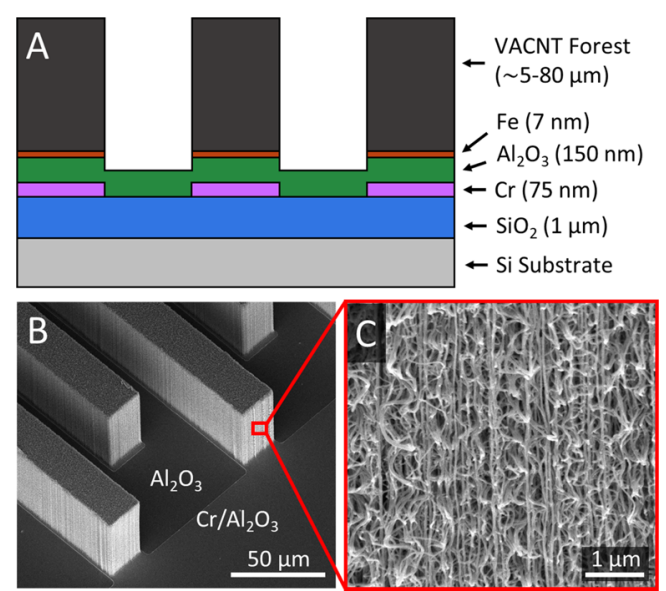

Figure 2. (A) Schematic of layers used to fabricate the VACNT sensor architecture: $\mathrm{Si}, \mathrm{SiO}_{2}, \mathrm{Cr}, \mathrm{Al}_{2} \mathrm{O}_{3}, \mathrm{Fe}$, and VACNTs. Scanning electron microscopy (SEM) images of (B) 3D VACNT electrodes and $(\mathrm{C})$ magnified VACNTs, showing the porous nature of an electrode.

silicon $(\mathrm{Si})$ wafer had patterned $\mathrm{Cr}$ and patterned iron $(\mathrm{Fe})$ separated by the insulating $\mathrm{Al}_{2} \mathrm{O}_{3}$ layer. Planar $\mathrm{Cr}$ electrical leads underneath $\mathrm{Al}_{2} \mathrm{O}_{3}$ can be seen in Figure $2 \mathrm{~B}$ as the lighter region on the substrate. VACNTs were grown by chemical vapor deposition (CVD) similar to previously published protocols. $^{30,31}$ After VACNT growth, amorphous carbon was added to strengthen the VACNTs and create mechanically sturdy, porous microstructures (see Figure $2 \mathrm{~B}, \mathrm{C}$ ). It should be noted that while VACNTs are generally vertically aligned, they are not perfectly straight and often exhibit some waviness (see Figure 2C) that is possibly due to edge effects caused by the iron catalyst and general strain on the VACNTs during growth and carbon infiltration. ${ }^{32,33}$ Additional scanning electron microscopy (SEM) images showing magnified views of the VACNTs are found in Figure S1 (Supporting Information).

Carbon-coated VACNTs are slightly hydrophobic after they are grown. An oxygen plasma etch was used to remove the amorphous carbon in between electrodes and also served to change the VACNTs from hydrophobic to hydrophilic. ${ }^{31}$ This hydrophilicity is important to ensure that the solution is wetting the entire electrode and to promote close contact between the molecules in solution and the electrode surface.

VACNT electrode heights were measured using optical profilometry. CVD growth times of 5, 30, and $120 \mathrm{~s}$ corresponded with approximately 5, 20, and $80 \mu \mathrm{m}$ electrode heights, respectively. The resulting aspect ratios $\left(h / w_{\mathrm{g}}\right)$ of the VACNT electrodes ranged from 0.2 to 5.3. The aspect ratio was limited by the height to which VACNT forests could grow without tipping over, causing an electrical short between the electrodes. This limiting height was a function of the electrode width $\left(w_{\mathrm{e}}\right)$ and likely also influenced by the gap width $\left(w_{\mathrm{g}}\right)$; an $80 \mu \mathrm{m}$ height approached the upper limit for the smaller electrode design $\left(w_{\mathrm{e}}=20 \mu \mathrm{m}, w_{\mathrm{g}}=15 \mu \mathrm{m}\right)$. Taller electrodes could be made by increasing $w_{\mathrm{e}}$, but sensitivity may also be affected because of the increase of underutilized surface area within the electrode.

3.3. Cyclic Voltammetry (CV). By sweeping the applied potential, CV can give important information about the geometry and electroactivity of an electrode. Figure 3A shows cyclic voltammograms of VACNT electrodes of varying heights
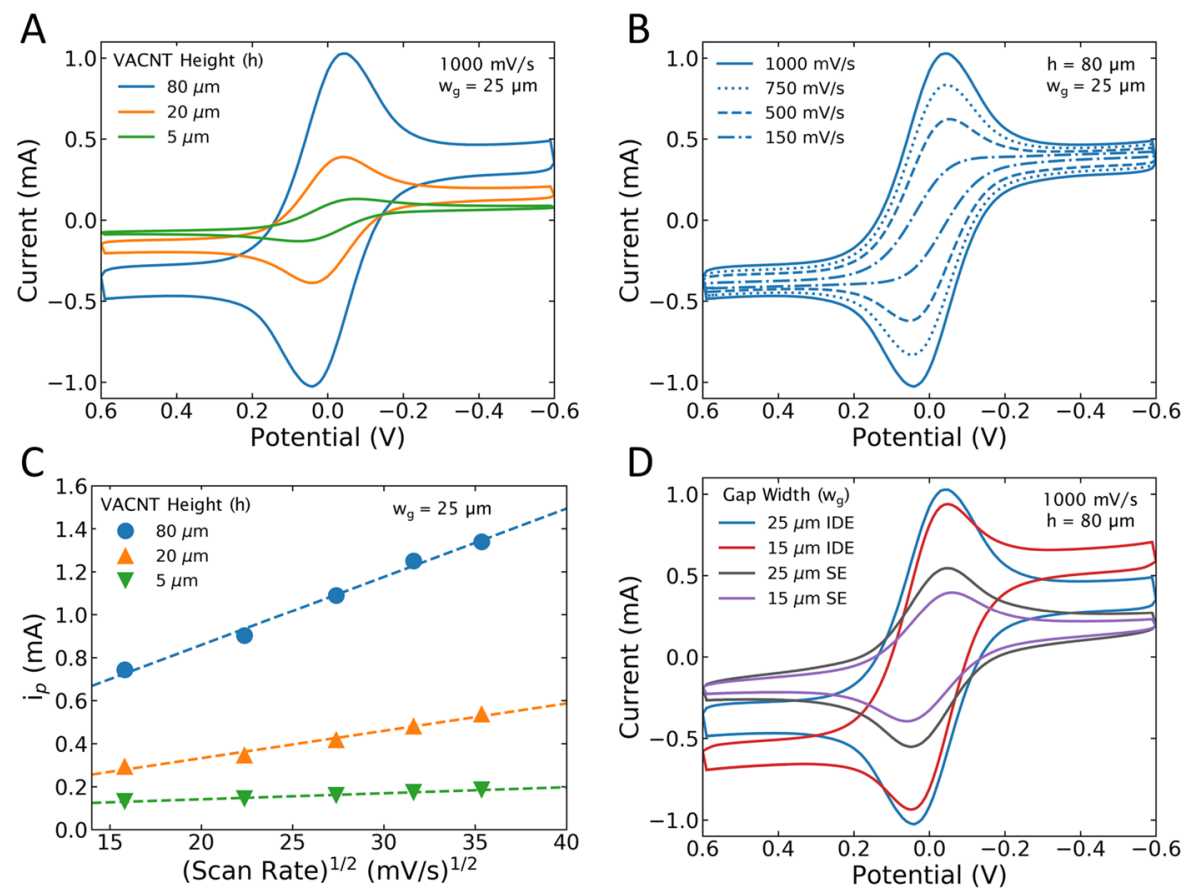

Figure 3. Cyclic voltammograms of VACNT electrodes in ferricyanide solution. (A) 80,20 , and $5 \mu \mathrm{m}$ tall VACNT IDEs $\left(w_{\mathrm{g}}=25 \mu \mathrm{m}\right)$ at a scan rate of $1000 \mathrm{mV} / \mathrm{s}$. (B) $80 \mu \mathrm{m}$ tall VACNT IDE $\left(w_{\mathrm{g}}=25 \mu \mathrm{m}\right)$ at scan rates of $1000,750,500$, and $150 \mathrm{mV} / \mathrm{s}$. (C) Peak current $i_{\mathrm{p}}$ with the square root of scan rate for 80,20 , and $5 \mu \mathrm{m}$ tall VACNTs. (D) $80 \mu \mathrm{m}$ tall VACNT IDEs and SEs with $w_{\mathrm{g}}$ values of 25 and $15 \mu \mathrm{m}$ at a scan rate of 1000 $\mathrm{mV} / \mathrm{s}$. 
A

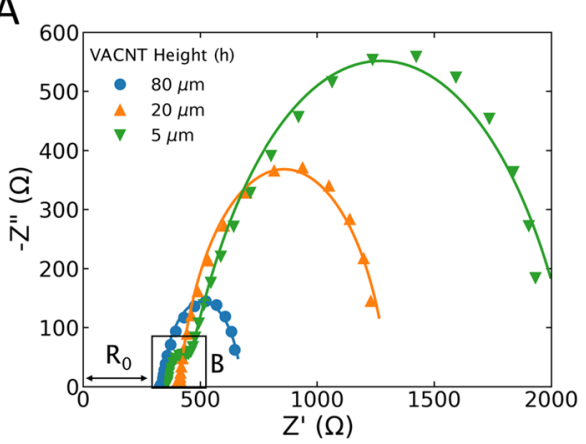

B

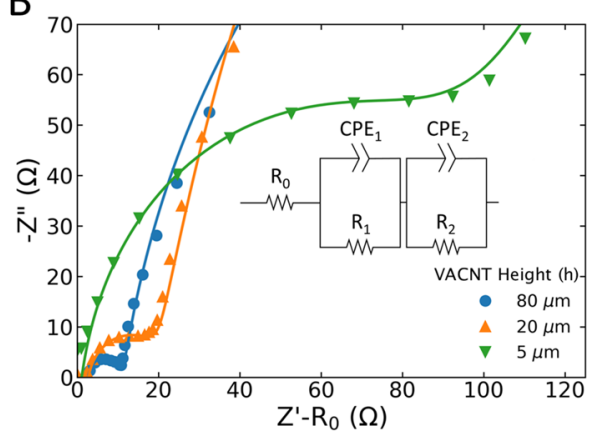

Figure 4. Experimental (markers) and simulated (lines) Nyquist plots of VACNT IDEs ( $\left.w_{\mathrm{g}}=25 \mu \mathrm{m}\right)$. (A) 80, 20, and $5 \mu \mathrm{m}$ tall VACNTs with no streptavidin/biotin. (B) Zoomed in view of Figure 4A normalized by $R_{\mathrm{o}}$, highlighting the trends for the first semicircle. Inset: equivalent circuit used to curve fit experimental data.

$(5,20$, and $80 \mu \mathrm{m})$ with $w_{\mathrm{g}}=25 \mu \mathrm{m}$ at a constant scan rate of $1000 \mathrm{mV} / \mathrm{s}$ in a static $50 \mu \mathrm{L}$ droplet of ferricyanide solution. The overall shape of the curves is typical for the single electron electrochemical reaction involving ferricyanide having anodic and cathodic peaks in the current. The peaks are caused by a balance between reaction kinetics and diffusion from the solution and are symmetric around $0 \mathrm{~V}$ because the reference is to a VACNT electrode and not a dedicated reference electrode such as $\mathrm{Ag} / \mathrm{AgCl}$.

Figure $3 \mathrm{~A}$ shows that VACNT height causes a significant difference in the peak current, with the $80 \mu \mathrm{m}$ peak current being about 7.2 times greater than $5 \mu \mathrm{m}$. The double layer capacitance also increases with increasing VACNT height, which is manifested by the increase in separation of the curves in the range from \pm 0.2 to $0.6 \mathrm{~V}$ in Figure 3A. The electroactive surface area of an electrode is linearly proportional to the peak current $\left(i_{\mathrm{p}}\right)$ as given by the Randles-Sevcik equation at $25{ }^{\circ} \mathrm{C}$ :

$$
i_{\mathrm{p}}=\left(2.69 \times 10^{5}\right) n^{3 / 2} A D^{1 / 2} C \nu^{1 / 2}
$$

where $n$ is the number of electrons transferred $(n=1), A$ is the electroactive area of the electrode (units of $\left.\mathrm{cm}^{2}\right), D$ is the diffusion coefficient of ferricyanide $\left(7.2 \times 10^{-6} \mathrm{~cm}^{2} / \mathrm{s}\right), C$ is the ferricyanide concentration $\left(5 \mathrm{mM}\right.$ or $\left.5 \times 10^{-6} \mathrm{~mol} / \mathrm{cm}^{3}\right)$, and $\nu$ is the scan rate $(0.125$ to $1.25 \mathrm{~V} / \mathrm{s})$. The only variable that is not well defined is the electrode surface area. The $2 \mathrm{D}$ geometric area of the electrode pattern is known to be 0.022 $\mathrm{cm}^{2}\left(30 L w_{e}\right)$, but the exact surface area of the electrode is not known because of the porous nature of the VACNTs. The calculated electroactive surface areas for the 80,20 , and $5 \mu \mathrm{m}$ tall VACNTs were $0.33,0.13$, and $0.046 \mathrm{~cm}^{2}$, respectively. Thus, the electroactive surface area of the $80 \mu \mathrm{m}$ tall electrode was 15 times larger than the $2 \mathrm{D}$ geometric area, with the actual surface area likely being even higher because of the electrode porosity.

The peak current increased with scan rate, as can be seen in Figure $3 \mathrm{~B}$ for $80 \mu \mathrm{m}$ tall VACNTs $\left(w_{\mathrm{g}}=25 \mu \mathrm{m}\right)$. It is interesting to note that the shape of the $\mathrm{CV}$ curve transitions from prominent peaks at high scan rates to a more sigmoidal shape with no peak at the lowest scan rate. This occurs because the electrodes are close enough together to cause a diffusionlimited scenario at slower scan rates, typical of microelectrode arrays. The peak-to-peak potential separation was about 85 $\mathrm{mV}$, which is close to the ideal Nernstian peak separation of 59 $\mathrm{mV}$. The peak-to-peak separation for other CNT-based electrochemical sensors is typically reported at about $80-100$
$\mathrm{mV} .{ }^{34,35}$ According to eq 1 , the peak current should be linearly proportional to the square root of scan rate, which is plotted in Figure $3 \mathrm{C}$ for the three different VACNT heights. This linearity, combined with the fact that the peak-to-peak potential separation did not increase with increasing scan rate (see Figure 3B), confirms that the VACNT electrode and the electrochemical reaction experience fast, reversible kinetic behavior. $^{36}$

Figure 3D shows cyclic voltammograms of various $80 \mu \mathrm{m}$ tall VACNT electrode designs. The blue curve is the same IDE geometry shown in Figure 3A,B, with $w_{\mathrm{g}}=25 \mu \mathrm{m}$. An IDE with $w_{\mathrm{g}}=15 \mu \mathrm{m}$ (red curve) exhibited a larger peak current than the $w_{\mathrm{g}}=25 \mu \mathrm{m}$ IDE (relative to the baseline scan current). Thus, the $15 \mu \mathrm{m}$ IDE had the highest electroactive surface area at $0.36 \mathrm{~cm}^{2}$ while having a smaller $2 \mathrm{D}$ area of $0.018 \mathrm{~cm}^{2}$, resulting in a 20 times increase in the electroactive surface area relative to a planar IDE. The peak currents for the SE curves (gray and purple) in Figure 3D are significantly lower than the IDEs. Even though the geometric dimensions and actual surface areas are the same for the IDE and SE configurations, the long winding electrodes in the SE configuration appears to reduce the effectiveness of the available area, perhaps because of the ohmic resistance along the lengthy meandering electrode. This reduced effectiveness of the SE surface area is opposite to the finding that SEs improve the performance of capacitive sensors, ${ }^{20}$ suggesting that SEs may not be as suitable for impedimetric sensors as they were for capacitive sensors. Additional $\mathrm{CV}$ data that shows the effect of scan rate on each geometry is found in Figure S2 (Supporting Information).

3.4. EIS Characterization. The IDE geometry was characterized further by EIS because it had a better electrochemical response than the SE geometry according to $\mathrm{CV}$ measurements. A fixed $w_{\mathrm{g}}=25 \mu \mathrm{m}$ was selected to assess variation in EIS measurements according to IDE height, as this geometry gave a higher yield of good VACNT growths compared to $w_{\mathrm{g}}=15 \mu \mathrm{m}$ IDEs. EIS measurements for the SE geometry and for $w_{\mathrm{g}}=15 \mu \mathrm{m}$ electrodes are discussed in Section 3.7.

Nyquist plots are a convenient way to show the results from EIS, where imaginary impedance $\left(-Z^{\prime \prime}\right)$ is plotted with respect to real impedance $\left(Z^{\prime}\right)$ over a range of frequencies. Nyquist plots for 80,20 , and $5 \mu \mathrm{m}$ tall IDEs $\left(w_{\mathrm{g}}=25 \mu \mathrm{m}\right)$ are shown in Figure 4A, where the impact of VACNT height on the baseline EIS measurement is evident by the change in magnitude of the semicircular curves. The impedance was much larger for the 5 
$\mu \mathrm{m}$ tall VACNTs than for the $80 \mu \mathrm{m}$ tall VACNTs, an observation that correlates well with the larger current observed from the $\mathrm{CV}$ data (Figure $3 \mathrm{~A}$ ) as a result of the higher surface area. Figure $4 \mathrm{~B}$ is a zoomed in view of the bottom left of the large semicircles from Figure 4A to highlight the second, smaller semicircular curve. These smaller semicircles follow the same trend as the larger semicircles, where the impedance of the $5 \mu \mathrm{m}$ tall VACNTs was larger than the impedance of the $80 \mu \mathrm{m}$ tall VACNTs. The data markers in the Nyquist plots in Figure 4 are the measured experimental EIS results for VACNT IDEs taken after EDC/NHS chemical activation but before the addition of streptavidin or biotin.

The inset of Figure 4B shows an equivalent electrical circuit that represents the observed behavior. Each semicircular shape in the Nyquist plot is representative of a resistance-capacitor time constant; as observed in Figure 4, two time constants are necessary to describe the sensor behavior. The equivalent circuit uses constant phase elements (CPEs) in place of capacitors, because the semicircles are depressed, which is more apparent when the axes of the Nyquist plot have an equivalent scale (see Figure $S 3$ in the Supporting Information). The location of resistance $R_{0}$ is shown in Figure $4 \mathrm{~A}$ and represents of the resistance in the $\mathrm{Cr}$ electrical leads. As $R_{0}$ varied between samples and between electrical probe positioning for each test, Nyquist plots were biased by subtracting out this resistance to so that the results could be presented clearly (see $x$ axis in Figure $4 B$ ). The first time constant $\left(C P E_{1} / R_{1}\right.$, small semicircle) is observed at higher frequencies (approximately $70-10,000 \mathrm{~Hz}$ ) and is associated with ferricyanide kinetics at the VACNT electrode surface, including charge transfer resistance and double layer capacitance. The second time constant $\left(\mathrm{CPE}_{2} / \mathrm{R}_{2}\right.$, large semicircle $)$ is observed at lower frequencies (approximately $0.1-70 \mathrm{~Hz}$ ) and is related to the diffusion of ferricyanide to and from the VACNT electrode surface. The IDE geometry has finite diffusion between the electrodes, causing the shape of the impedance in the Nyquist plot to bend in an arc instead extend linearly as is the case in semi-infinite diffusion. ${ }^{37}$ Although a traditional Warburg element does not apply (semi-infinite only), the diffusion component could also be modeled by an open finite-length Warburg element or a finite-length CPE element, with CPEs being able to better fit experimental data as shown in Figure S4 in the Supporting Information. ${ }^{37}$ A similar equivalent circuit with two $\mathrm{CPE} / \mathrm{R}$ pairs is also used to model lithium-ion batteries, where the first time constant is attributed to an interfacial layer, and the second time constant is attributed to charge transfer resistance and double layer capacitance (Figure S4 in the Supporting Information). ${ }^{38}$ Our previous VACNT sensor had a large semicircle at high frequencies because of the capacitance along the VACNT electrical leads, ${ }^{13}$ but that feature was not observed when using underlying $\mathrm{Cr}$ electrical leads. The equivalent circuit in Figure 4B was used to curve fit the experimental EIS data with EIS Spectrum Analyzer software, and the simulated results are plotted as the solid lines in Figure 4. The values of curve fit equivalent circuit elements for VACNT IDE heights of 5, 20, and $80 \mu \mathrm{m}$ are given in Table S1 (Supporting Information). The simulated results matched the experimental results with reasonable accuracy.

3.5. Functionalization of F-SA and Binding of FBiotin. It has previously been shown by Raman spectroscopy that VACNTs coated with amorphous carbon have a high number of surface defects, an attribute favorable for increasing the number of sites where proteins can bind and increasing charge transfer during electrochemical measurements. ${ }^{13}$ Due to the addition of amorphous carbon to the VACNTs, defect sites that host carboxyl groups are present throughout the VACNT electrode surface. These carboxyl groups were activated by EDC/NHS chemistry to allow fluorescently tagged streptavidin (F-SA) to covalently bond to the VACNTs (see Scheme 1). Fluorescently tagged biotin (F-biotin) was then bound to the F-SA, as biotin binds to streptavidin in one of the strongest known noncovalent bonds between a protein and a ligand. ${ }^{29}$

Because the fluorescent dye only has $2 \%$ of the mass of the streptavidin, the impedance and binding results are expected to be similar to unlabeled streptavidin. On the other hand, the molecular weight of the F-biotin is $921.5 \mathrm{~g} / \mathrm{mol}$, about 3.8 times larger than unlabeled biotin at $244.3 \mathrm{~g} / \mathrm{mol}$, but still significantly smaller than streptavidin at $53,000 \mathrm{~g} / \mathrm{mol}$. F-biotin and F-SA have diameters of approximately 1.2 and $5 \mathrm{~nm}$, respectfully. ${ }^{39}$ Thus, F-biotin has a volume that is about 58 times smaller than F-SA.

Fluorescently tagged molecules were used to enable a qualitative confirmation of the coverage of streptavidin and biotin on the VACNTs by fluorescence microscopy. Figure 5A
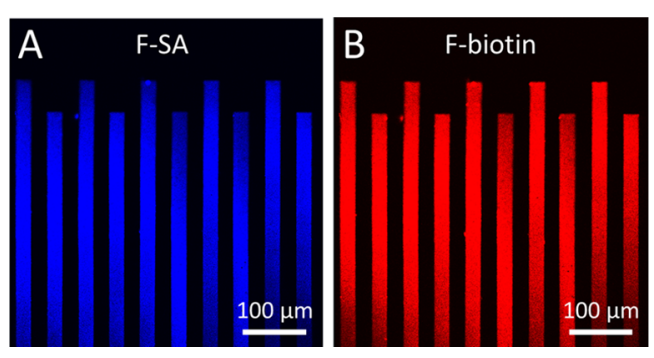

Figure 5. Fluorescence microscopy images confirming good coverage of (A) $500 \mu \mathrm{g} / \mathrm{mL}$ fluorescently tagged streptavidin (F-SA, blue) bound to $80 \mu \mathrm{m}$ tall VACNT IDEs and (B) $100 \mu \mathrm{g} / \mathrm{mL}$ fluorescently tagged biotin (F-biotin, red) bound to F-SA. Note that F-SA and Fbiotin are present in both images (same sensor), but fluorescence is at different wavelengths.

shows the blue fluorescence of F-SA on VACNT IDE electrode fingers, and Figure 5 shows the red fluorescence of F-biotin bound to the F-SA on the same device. The protein-ligand binding appears efficient across the entire surface of the VACNTs as displayed in the top down view shown in Figure 5 for the binding performed by a static droplet on the VACNTs with a concentration of $500 \mathrm{~F}-\mathrm{SA}$ and $100 \mu \mathrm{g} / \mathrm{mL}$ F-biotin. It should be noted that there was also some coverage on the substrate, but this appears black in Figure 5 images because it is out of the microscope focal plane.

While the functionalization and binding steps for the sensor were carried out with a static droplet on the VACNTs, the sensors are designed with an underlying $\mathrm{Cr}$ layer to allow for potential future integration in a microfluidic channel to increase mass transfer of the molecules to the electrode surface. Our previous flow-through VACNT electrodes for hydrogen peroxide ${ }^{30}$ and glucose ${ }^{40}$ detection showed that flowing conditions greatly improved amperometric signals. Microfluidic IDE sensors have also been shown to be more sensitive than static IDE sensors. ${ }^{25}$ The exposed Cr leads provide the means for electrical contact within the plane of the sensor substrate to allow a $3 \mathrm{D}$-printed microfluidic channel to be clamped over the sensor (see also Figure S5, Supporting Information). Although fluorescence showed better coverage 

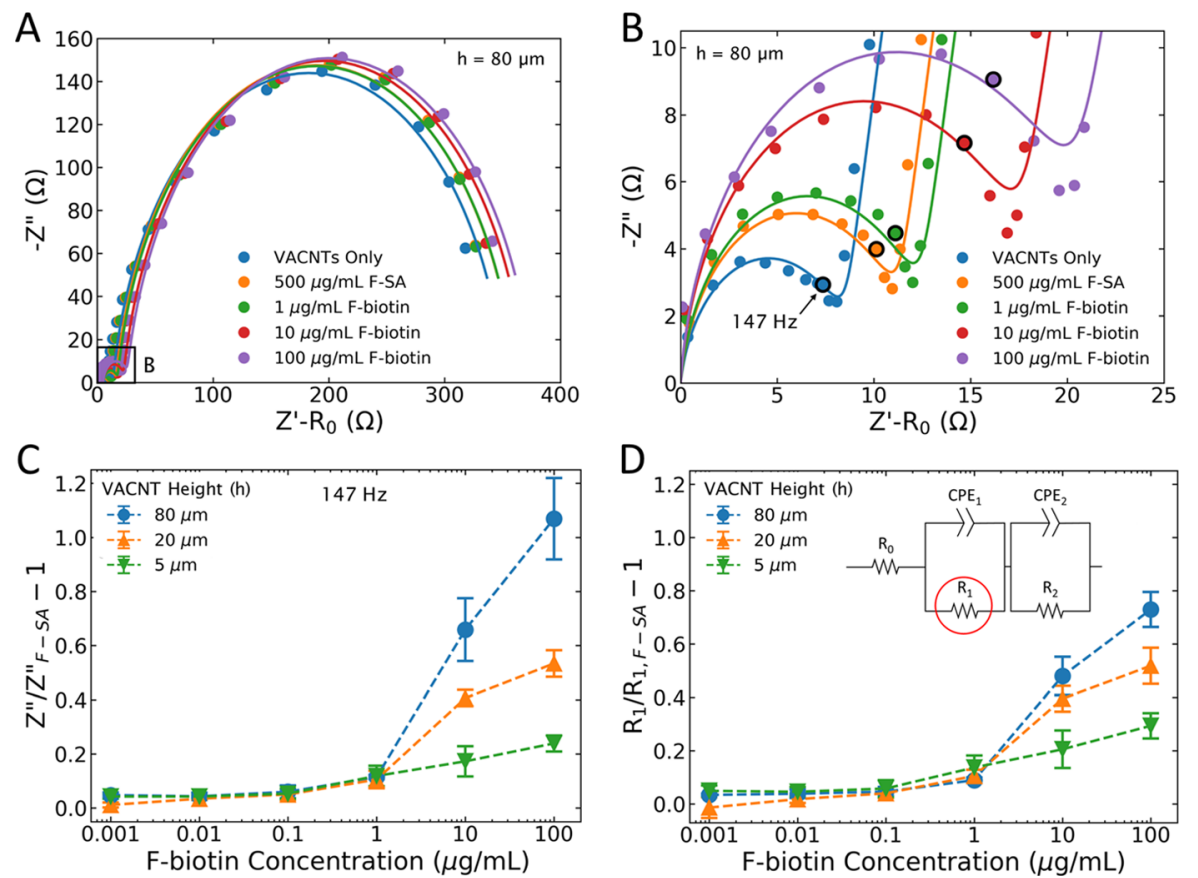

Figure 6. (A) Experimental (markers) and simulated (lines) Nyquist plots of $80 \mu \mathrm{m}$ tall VACNT IDEs $\left(w_{\mathrm{g}}=25 \mu \mathrm{m}\right)$ with subsequent addition of $500 \mu \mathrm{g} / \mathrm{mL}$ F-SA and 1, 10, and $100 \mu \mathrm{g} / \mathrm{mL}$ F-biotin. (B) Zoomed in view of the first semicircle of Figure 7A, with $147 \mathrm{~Hz}$ marked with a black outline. Relative change in (C) imaginary impedance at $147 \mathrm{~Hz}$ and (D) $R_{1}$ resistance from an equivalent circuit as a function of F-biotin concentration for 80,20 , and $5 \mu \mathrm{m}$ tall VACNT IDEs. Error bars represent the standard deviation for three different sensors.

using the microchannel (Figure S6, Supporting Information), initial electrochemical tests with the microfluidic channel demonstrated little improvement over static F-SA and F-biotin binding. Thus, static tests were chosen for experimental simplicity. However, the proof of concept was successful, such that the sensor could be incorporated into a microchannel. There likely exist applications where the microfluidic channel would be beneficial. We note that this integration can be achieved without loss in signal strength although further investigation is likely needed for optimal microfluidic conditions in a given application.

3.6. Biotin Sensing with IDEs. The impedance change associated with F-SA and F-biotin binding to a sample sensor surface is shown in Figure 6A,B for an $80 \mu \mathrm{m}$ tall VACNT IDE $\left(w_{\mathrm{g}}=25 \mu \mathrm{m}\right)$. Note that IDEs with $w_{\mathrm{g}}=25 \mu \mathrm{m}$ are used for Fbiotin sensing in this section, and F-biotin sensing of other geometries are compared in Section 3.7. As shown in Figure $6 \mathrm{~A}$, there was a slight change observed in the resistance of the diffusion-driven large semicircles when adding F-SA and Fbiotin to the VACNTs (1.8\% F-SA, 3.5\% F-SA + F-biotin), but there was a much larger change observed in the resistance of the kinetics-driven small semicircles shown in Figure 6B (32\% F-SA, $138 \%$ F-SA + F-biotin). Thus, it is advantageous to focus on the changes that occur at the higher frequencies associated with the kinetics at the electrode surface.

Two options were considered for monitoring changes in the small semicircle in response to molecules bound to the surface. The first and more direct option is to use impedance data measured at a single frequency. Specifically, the imaginary component of impedance $Z^{\prime \prime}$ exhibited the largest relative change when F-biotin was bound to the VACNTs. Figure 6C shows the relative change in imaginary impedance at $147 \mathrm{~Hz}$ for 80,20 , and $5 \mu \mathrm{m}$ tall VACNTs for different concentrations of F-biotin, where the percent change is measured relative to the imaginary impedance at $147 \mathrm{~Hz}$ after functionalizing with
F-SA. The percent change in imaginary impedance was calculated across all measured frequencies, and it was determined that $147 \mathrm{~Hz}$ gave the highest signal for all heights (see also the Bode plot in Figure S7 in the Supporting Information). The location of the $147 \mathrm{~Hz}$ impedance is on the right side of the small semicircle, as shown by the data markers outlined in black in Figure 6B. The second option for monitoring changes is shown in Figure $6 \mathrm{D}$, where the relative change in charge transfer resistance $R_{1}$ from the equivalent circuit model is plotted for different F-biotin concentration for 80,20 , and $5 \mu \mathrm{m}$ tall VACNTs. Because this method uses an equivalent circuit resistance, the fitted parameter accounts for all the measured frequencies instead of just a single frequency. The single frequency method and the equivalent circuit method with curve fit parameters give relative changes that follow a similar trend and are comparable in magnitude. The relative change in imaginary impedance at $147 \mathrm{~Hz}$ generally gave a higher signal than the change in $R_{1}$ resistance; therefore, the results for all subsequent geometries are reported only in terms of relative imaginary impedance change. The data in Figure $6 \mathrm{C}, \mathrm{D}$ represent the average of three sensors, with the error bars indicating the standard deviation.

In Figure 6C,D, it can be seen that the changes in impedance were small at lower concentrations of F-biotin (1 $\mathrm{ng} / \mathrm{mL}$ to $1 \mu \mathrm{g} / \mathrm{mL}$ ), and then there was a sudden spike in impedance at $10 \mu \mathrm{g} / \mathrm{mL}$ F-biotin, potentially leading to two linear sensing regions. While the exact cause of this phenomenon is unknown, it is possible that higher concentrations of F-biotin allow the molecules to better penetrate the porous VACNT electrode because of a higher concentration gradient, thus utilizing more of the surface area in between VACNTs. The $80 \mu \mathrm{m}$ VACNTs provided an average change in an imaginary impedance of $7 \%$ from $0.001-$ $1 \mu \mathrm{g} / \mathrm{mL}$ F-biotin and an average change of $95 \%$ from $1-100$ $\mu \mathrm{g} / \mathrm{mL}$ F-biotin. The overall maximum change in imaginary 

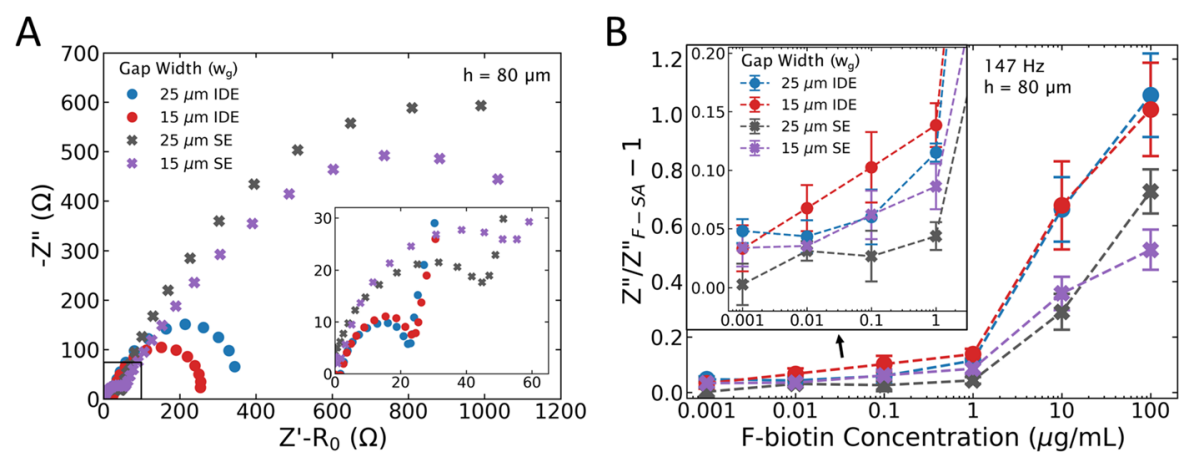

Figure 7. (A) Nyquist plots for $80 \mu \mathrm{m}$ tall VACNT IDEs and SEs with $w_{\mathrm{g}}$ values of 15 and $25 \mu \mathrm{m}$. Inset shows a zoomed in view of smaller semicircles near the origin. (B) Relative change in imaginary impedance $Z^{\prime \prime}$ at $147 \mathrm{~Hz}$ as a function of F-biotin concentration for $80 \mu \mathrm{m}$ tall VACNT IDEs and SEs with $w_{\mathrm{g}}$ values of 15 and $25 \mu \mathrm{m}$. Inset shows close up of F-biotin concentrations from $1 \mathrm{ng} / \mathrm{mL}$ to $1 \mu \mathrm{g} / \mathrm{mL}$. Error bars represent the standard deviation for three different sensors.

impedance was $107 \%$ with $100 \mu \mathrm{g} / \mathrm{mL}$ F-biotin (relative to FSA). The tall VACNT IDEs $(80 \mu \mathrm{m})$ gave 4.3 times the signal of the $5 \mu \mathrm{m}$ VACNT IDEs, likely due to the additional surface area and small baseline impedance of the bare VACNTs (see Figure 4B).

3.7. Electrode Geometry Impact on Sensitivity. EIS data shown up to this point have been for IDEs with $w_{\mathrm{g}}=25$ $\mu \mathrm{m}$. The SE design shown in Figure $1 \mathrm{~B}$ was explored as a potential alternative design to the more traditional IDE, as it has previously been shown to exhibit a large signal change associated with capacitance. ${ }^{20}$ Electrodes with $w_{\mathrm{g}}=15 \mu \mathrm{m}$ were also tested, as closely spaced electrodes generally provide better sensitivity.

Figure 7A shows Nyquist plots for $80 \mu \mathrm{m}$ tall IDEs and SEs with $w_{\mathrm{g}}$ values of 25 and $15 \mu \mathrm{m}$ having F-SA and F-biotin bound to the VACNTs. The impedance was significantly larger for the SEs, with the large semicircle impedance related to diffusion being about five times larger than that for the IDEs. The resistance for both the IDE and SE with $w_{\mathrm{g}}=15 \mu \mathrm{m}$ was reduced relative to $w_{\mathrm{g}}=25 \mu \mathrm{m}$, confirming a shorter diffusion length with a smaller gap between electrodes. The inset of Figure 7A shows that the small semicircle resistances related to kinetics for the SEs were larger in magnitude than the resistance of IDEs.

The relative change in imaginary impedance with F-biotin concentration is shown in Figure $7 \mathrm{~B}$ for the same geometric configurations shown in Figure 7A. The tracking frequency that provided that largest change in imaginary impedance remained at $147 \mathrm{~Hz}$ for both IDEs and SEs. A very similar trend was observed for each geometry, with a large change in impedance observed with increasing concentration above 1 $\mu \mathrm{g} / \mathrm{mL}$ F-biotin. The results were repeatable for the different geometries tested, as the standard deviation between sensors of the same geometry (error bars in Figure 7B) was relatively small. On average, the IDEs were found to be about 1.6 times more sensitive than SEs. The inset in Figure 7B shows that the impedance still increased with increasing F-biotin concentrations below $1 \mu \mathrm{g} / \mathrm{mL}$, with an IDE of $w_{\mathrm{g}}=15 \mu \mathrm{m}$ having the highest sensitivity and also being the most linear among the geometries tested. Thus, the $80 \mu \mathrm{m}$ tall VACNT IDE with $w_{\mathrm{g}}=$ $15 \mu \mathrm{m}$ had a linear sensing region from $1 \mathrm{ng} / \mathrm{mL}$ to $1 \mu \mathrm{g} / \mathrm{mL}$ and a secondary linear sensing region from 1 to $100 \mu \mathrm{g} / \mathrm{mL}$. Two different linear ranges for detection have been reported by others for amperometric and impedimetric sensing, but the slope typically is more gradual at higher concentrations, as the electrode becomes saturated with an analyte. The $80 \mu \mathrm{m}$ tall
IDE with $w_{\mathrm{g}}=15 \mu \mathrm{m}$ had a detection limit of $1 \mathrm{ng} / \mathrm{mL}$, which is similar to the detection limit of other biotin sensors reported in the literature. ${ }^{41,42}$

Modest changes in the sensing signal were observed at Fbiotin concentrations below $1 \mu \mathrm{g} / \mathrm{mL}$. One potential reason is that F-biotin is a small molecule, much smaller than proteins, so that the change with concentration is likely smaller than for larger protein molecules. The VACNT IDEs were previously shown to have a limit of detection in the picomolar range when detecting oral cancer biomarkers, ${ }^{13}$ instead of in the nanomolar range that is here observed with F-biotin. The exploration of the effect of molecular size, as well as the investigation of nonspecific binding, are potential extensions of this work. However, the oral cancer VACNT biosensor has already shown that impedance-based sensing is possible in complex saliva supernatant with VACNT electrodes and showed minimal nonspecific binding from bovine serum albumin (BSA) protein. ${ }^{13}$ The main goal of the current work is to examine the influence of height for very tall VACNT electrodes and compare the geometric influence of these devices for a representative protein model.

\section{CONCLUSIONS}

This work has characterized porous, 3D VACNT electrodes for impedance-based biosensing. Electrode height was shown to have a large impact on CV and EIS curves, with tall $80 \mu \mathrm{m}$ VACNTs having the highest calculated electroactive surface area (approximately 15 times the $2 \mathrm{D}$ geometric area) and thus resulting in the smallest baseline kinetic resistance as seen through the Nyquist plots. The representative protein streptavidin was functionalized onto VACNT electrodes for detection of biotin, and good coverage was confirmed by fluorescence microscopy. EIS was used to measure the change in impedance across electrodes for different biotin concentrations. Two linear sensing regions are observed for all electrode geometries, and IDEs had higher sensitivity than SEs. The relative change in imaginary impedance from F-biotin was shown to be as high as $107 \%$ for IDEs. The geometry with the highest sensitivity and the most linear sensing region was an 80 $\mu \mathrm{m}$ tall VACNT IDE with a gap width of $15 \mu \mathrm{m}$, enabling a limit of detection of $1 \mathrm{ng} / \mathrm{mL}$ F-biotin.

Although this biosensing platform is shown with streptavidin and biotin, it could be extended to other proteins, antibodies, viruses, and bacteria depending on surface functionalization. By having multiple sensors in series, it would be possible to simultaneously detect many different target analytes. It is 
expected that this structure and sensing approach will be viable for a range of electrochemical sensing, particularly in medical diagnostics.

\section{ASSOCIATED CONTENT}

\section{SI Supporting Information}

The Supporting Information is available free of charge at https://pubs.acs.org/doi/10.1021/acsanm.0c02121.

Additional SEM images, CV data, Nyquist plots of EIS data, values of equivalent circuit elements, images of a 3D-printed microfluidic channel, fluorescence microscopy images, and Bode plot (PDF)

\section{AUTHOR INFORMATION}

\section{Corresponding Author}

Brian D. Iverson - Department of Mechanical Engineering, Brigham Young University, Provo, Utah 84602, United States; ○ orcid.org/0000-0002-4592-3728; Email: bdiverson@ byu.edu

\section{Authors}

Benjamin J. Brownlee - Department of Mechanical Engineering, Brigham Young University, Provo, Utah 84602, United States

Jonathan C. Claussen - Department of Mechanical Engineering, Iowa State University, Ames, Iowa 50011, United

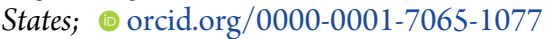

Complete contact information is available at: https://pubs.acs.org/10.1021/acsanm.0c02121

\section{Notes}

The authors declare no competing financial interest.

\section{ACKNOWLEDGMENTS}

The authors gratefully acknowledge support from the Utah NASA Space Grant Consortium as well as the Department of Mechanical Engineering and College of Engineering and Technology at Brigham Young University.

\section{REFERENCES}

(1) Andoy, N. M.; Filipiak, M. S.; Vetter, D.; Gutiérrez-Sanz, Ó.; Tarasov, A. Graphene-Based Electronic Immunosensor with Femtomolar Detection Limit in Whole Serum. Adv. Mater. Technol. 2018, 3, 1800186.

(2) Dhara, K.; Mahapatra, D. R. Electrochemical Nonenzymatic Sensing of Glucose Using Advanced Nanomaterials. Microchim. Acta 2018, 185, 49.

(3) Schröper, F.; Brüggemann, D.; Mourzina, Y.; Wolfrum, B.; Offenhäusser, A.; Mayer, D. Analyzing the electroactive surface of gold nanopillars by electrochemical methods for electrode miniaturization. Electrochim. Acta 2008, 53, 6265-6272.

(4) Tsentalovich, D. E.; Headrick, R. J.; Mirri, F.; Hao, J.; Behabtu, N.; Young, C. C.; Pasquali, M. Influence of Carbon Nanotube Characteristics on Macroscopic Fiber Properties. ACS Appl. Mater. Interfaces 2017, 9, 36189-36198.

(5) Huang, Q.; Lin, X.; Tong, L.; Tong, Q.-X. Graphene Quantum Dots/Multiwalled Carbon Nanotubes Composite-Based Electrochemical Sensor for Detecting Dopamine Release from Living Cells. ACS Sustainable Chem. Eng. 2020, 8, 1644-1650.

(6) Mynttinen, E.; Wester, N.; Lilius, T.; Kalso, E.; Mikladal, B.; Varjos, I.; Sainio, S.; Jiang, H.; Kauppinen, E. I.; Koskinen, J.; Laurila, T. Electrochemical Detection of Oxycodone and Its Main Metabolites with Nafion-Coated Single-Walled Carbon Nanotube Electrodes. Anal. Chem. 2020, 92, 8218-8227.
(7) Kumar, S.; Rani, R.; Dilbaghi, N.; Tankeshwar, K.; Kim, K.-H. Carbon Nanotubes: A Novel Material for Multifaceted Applications in Human Healthcare. Chem. Soc. Rev. 2017, 46, 158-196.

(8) Ognjanović, M.; Stanković, D. M.; Jović, M.; Krstić, M. P.; Lesch, A.; Girault, H. H.; Antić, B. Inkjet-Printed Carbon Nanotube Electrodes Modified with Dimercaptosuccinic Acid-Capped $\mathrm{Fe}_{3} \mathrm{O}_{4}$ Nanoparticles on Reduced Graphene Oxide Nanosheets for SingleDrop Determination of Trifluoperazine. ACS Applied Nano Mater. 2020, 3, 4654-4662.

(9) Valentine, C. J.; Takagishi, K.; Umezu, S.; Daly, R.; De Volder, M. Paper-Based Electrochemical Sensors Using Paper as a Scaffold to Create Porous Carbon Nanotube Electrodes. ACS Appl. Mater. Interfaces 2020, 12, 30680-30685.

(10) Zhu, P.; Ou, H.; Kuang, Y.; Hao, L.; Diao, J.; Chen, G. Cellulose Nanofiber/Carbon Nanotube Dual Network-Enabled Humidity Sensor with High Sensitivity and Durability. ACS Appl. Mater. Interfaces 2020, 12, 33229-33238.

(11) Ahmad, R.; Tripathy, N.; Ahn, M.-S.; Hahn, Y.-B. Solution Process Synthesis of High Aspect Ratio ZnO Nanorods on Electrode Surface for Sensitive Electrochemical Detection of Uric Acid. Sci. Rep. 2017, 7, 46475

(12) Kim, D.; Herr, A. E. Protein immobilization techniques for microfluidic assays. Biomicrofluidics 2013, 7, No. 041501.

(13) Ding, S.; Das, S. R.; Brownlee, B. J.; Parate, K.; Davis, T. M.; Stromberg, L. R.; Chan, E. K. L.; Katz, J.; Iverson, B. D.; Claussen, J. C. CIP2A Immunosensor Comprised of Vertically-aligned Carbon Nanotube Interdigitated Electrodes Towards Point-of-Care Oral Cancer Screening. Biosens. Bioelectron. 2018, 117, 68-74.

(14) Zaidi, S. A.; Shin, J. H. Recent Developments in Nanostructure Based Electrochemical Glucose Sensors. Talanta 2016, 149, 30-42.

(15) Varshney, M.; Li, Y.; Srinivasan, B.; Tung, S. A label-free, microfluidics and interdigitated array microelectrode-based impedance biosensor in combination with nanoparticles immunoseparation for detection of Escherichia coli O157:H7 in food samples. Sens. Actuators, B 2007, 128, 99-107.

(16) Daniels, J. S.; Pourmand, N. Label-Free Impedance Biosensors: Opportunities and Challenges. Electroanalysis 2007, 19, 1239-1257.

(17) Ding, S.; Mosher, C.; Lee, X. Y.; Das, S. R.; Cargill, A. A.; Tang, X.; Chen, B.; McLamore, E. S.; Gomes, C.; Hostetter, J. M.; Claussen, J. C. Rapid and Label-Free Detection of Interferon Gamma via an Electrochemical Aptasensor Comprising a Ternary Surface Monolayer on a Gold Interdigitated Electrode Array. ACS Sens. 2017, 2, 210217.

(18) Lequin, R. M. Enzyme Immunoassay (EIA)/Enzyme-Linked Immunosorbent Assay (ELISA). Clin. Chem. 2005, 51, 2415-2418.

(19) Chikkaveeraiah, B. V.; Bhirde, A. A.; Morgan, N. Y.; Eden, H. S.; Chen, X. Electrochemical Immunosensors for Detection of Cancer Protein Biomarkers. ACS Nano 2012, 6, 6546-6561.

(20) Rivadeneyra, A.; Fernández-Salmerón, J.; Banqueri, J.; LópezVillanueva, J. A.; Capitan-Vallvey, L. F.; Palma, A. J. A novel electrode structure compared with interdigitated electrodes as capacitive sensor. Sens. Actuators, B 2014, 204, 552-560.

(21) Sun, J.; Zheng, M.; Luo, Y.; Yu, Z. Three-dimensional detached serpentine flow field design for redox flow batteries. J. Power Sources 2019, 428, 136-145.

(22) Chiou, Y.-D.; Tsai, D.-S.; Lam, H. H.; Chang, C.-h.; Lee, K.-Y.; Huang, Y.-S. Cycle stability of the electrochemical capacitors patterned with vertically aligned carbon nanotubes in an LiPF6based electrolyte. Nanoscale 2013, 5, 8122-8129.

(23) Hsia, B.; Marschewski, J.; Wang, S.; In, J. B.; Carraro, C.; Poulikakos, D.; Grigoropoulos, C. P.; Maboudian, R. Highly flexible, all solid-state micro-supercapacitors from vertically aligned carbon nanotubes. Nanotechnology 2014, 25, No. 055401.

(24) Liu, C.-C.; Tsai, D.-S.; Chung, W.-H.; Li, K.-W.; Lee, K.-Y.; Huang, Y.-S. Electrochemical micro-capacitors of patterned electrodes loaded with manganese oxide and carbon nanotubes. J. Power Sources 2011, 196, 5761-5768.

(25) Manczak, R.; Fouet, M.; Courson, R.; Fabre, P.-L.; Montrose, A.; Sudor, J.; Gué, A.-M.; Reybier, K. Improved on-chip impedimetric 
immuno-detection of subpopulations of cells toward single-cell resolution. Sens. Actuators, B 2016, 230, 825-831.

(26) Sugime, H.; Ushiyama, T.; Nishimura, K.; Ohno, Y.; Noda, S. An interdigitated electrode with dense carbon nanotube forests on conductive supports for electrochemical biosensors. Analyst 2018, 143, 3635-3642.

(27) Chen, G.; Dodson, B.; Hedges, D. M.; Steffensen, S. C.; Harb, J. N.; Puleo, C.; Galligan, C.; Ashe, J.; Vanfleet, R. R.; Davis, R. C. Fabrication of High Aspect Ratio Millimeter-Tall Free-Standing Carbon Nanotube-Based Microelectrode Arrays. ACS Biomater. Sci. Eng. 2018, 4, 1900-1907.

(28) Deng, J.; Jiang, X. Advances in Reagents Storage and Release in Self-Contained Point-of-Care Devices. Adv. Mater. Technol. 2019, 4, 1800625.

(29) Diamandis, E. P.; Christopoulos, T. K. The biotin-(strept)avidin system: principles and applications in biotechnology. Clin. Chem. 1991, 37, 625-636.

(30) Brownlee, B. J.; Marr, K. M.; Claussen, J. C.; Iverson, B. D. Improving Sensitivity of Electrochemical Sensors with Convective Transport in Free-standing, Carbon Nanotube Structures. Sens. Actuators, B 2017, 246, 20-28.

(31) Marr, K. M.; Chen, B.; Mootz, E. J.; Geder, J.; Pruessner, M.; Melde, B. J.; Vanfleet, R. R.; Medintz, I. L.; Iverson, B. D.; Claussen, J. C. High Aspect Ratio Carbon Nanotube Membranes Decorated with Pt Nanoparticle Urchins for Micro Underwater Vehicle Propulsion via $\mathrm{H}_{2} \mathrm{O}_{2}$ Decomposition. ACS Nano 2015, 9, 7791-7803.

(32) Moulton, K.; Morrill, N. B.; Konneker, A. M.; Jensen, B. D.; Vanfleet, R. R.; Allred, D. D.; Davis, R. C. Effect of iron catalyst thickness on vertically aligned carbon nanotube forest straightness for CNT-MEMS. J. Micromech. Microeng. 2012, 22, No. 055004.

(33) Jahangiri, M. Systematic periodicity in waviness of vertically aligned carbon nanotubes explained by helical buckling. Nanotechnology 2017, 28, 375706.

(34) Koehne, J.; Li, J.; Cassell, A. M.; Chen, H.; Ye, Q.; Ng, H. T.; Han, J.; Meyyappan, M. The fabrication and electrochemical characterization of carbon nanotube nanoelectrode arrays. J. Mater. Chem. 2004, 14, 676-684.

(35) Kang, X.; Mai, Z.; Zou, X.; Cai, P.; Mo, J. A sensitive nonenzymatic glucose sensor in alkaline media with a copper nanocluster/multiwall carbon nanotube-modified glassy carbon electrode. Anal. Biochem. 2007, 363, 143-150.

(36) Elgrishi, N.; Rountree, K. J.; McCarthy, B. D.; Rountree, E. S.; Eisenhart, T. T.; Dempsey, J. L. A Practical Beginner's Guide to Cyclic Voltammetry. J. Chem. Educ. 2018, 95, 197-206.

(37) Lai, C.-Y.; Weng, J.-H.; Shih, W.-L.; Chen, L.-C.; Chou, C.-F.; Wei, P.-K. Diffusion impedance modeling for interdigitated array electrodes by conformal mapping and cylindrical finite length approximation. Electrochim. Acta 2019, 320, 134629.

(38) Choi, W.; Shin, H.-C.; Kim, J. M.; Choi, J.-Y.; Yoon, W.-S. Modeling and Applications of Electrochemical Impedance Spectroscopy (EIS) for Lithium-ion Batteries. J. Electrochem. Sci. Technol. 2020, 11, 1-13.

(39) Erickson, H. P. Size and Shape of Protein Molecules at the Nanometer Level Determined by Sedimentation, Gel Filtration, and Electron Microscopy. Biol. Proced. Online 2009, 11, 32.

(40) Brownlee, B. J.; Bahari, M.; Harb, J. N.; Claussen, J. C.; Iverson, B. D. Electrochemical Glucose Sensors Enhanced by Methyl Viologen and Vertically Aligned Carbon Nanotube Channels. ACS Appl. Mater. Interfaces 2018, 10, 28351-28360.

(41) Lee, S.-J.; Anandan, V.; Zhang, G. Electrochemical fabrication and evaluation of highly sensitive nanorod-modified electrodes for a biotin/avidin system. Biosens. Bioelectron. 2008, 23, 1117-1124.

(42) Buzid, A.; Hayes, P. E.; Glennon, J. D.; Luong, J. H. T. Captavidin as a regenerable biorecognition element on boron-doped diamond for biotin sensing. Anal. Chim. Acta 2019, 1059, 42-48. 